\title{
Guidelines: Evidenz als Dreh- und Angelpunkt*
}

Consensus means that lots of people believe collectively, what nobody believes individually. (Abba Eban)

\section{Thomas F. Lüscher ${ }^{a}$, Gilbert Abetel ${ }^{b}$}

a Prof. Dr. med., Direktor der Klinik für Kardiologie, HerzKreislaufZentrum, UniversitätsSpital Zürich. Der Autor ist gegenwärtig nicht Mitglied eines

Guidelines Committees, berät aber und ist Inhaber von Forschungsverträgen mit den meisten pharmazeutischen Firmen und Herstellern von medizinischen Geräten. Er ist zudem Editor-in-chief des European Heart Journals, welcher alle Guidelines der European Society of Cardiology publiziert, und Herausgeber des ESC Textbooks of Cardiovascular Medicine (Oxford University Press 2009), welches seine Empfehlungen auf diese Guidelines abstützt.

b Dr. med., Facharzt für Innere Medizin, Orbe

* Dieser Beitrag in der Serie «Zusammenarbeit ÄrzteschaftIndustrie» widerspiegelt die persönliche Meinung von Mitgliedern der Beratenden Kommission und ist nicht als offizielle Stellungnahme der Kommission oder der SAMW selbst zu verstehen.

\section{Korrespondenz:}

Prof. Thomas F. Lüscher UniversitätsSpital Zürich

Rämistrasse 100

CH-8091 Zürich

karlue@usz.unizh.ch

Dr Gilbert Abetel

Place du Marché 6

CH-1350 Orbe

Gilbert.Abetel@hin.ch
Seit es Menschen gibt, sind Ratschläge anderer wichtig - am Anfang war es der Rat der Ältesten, derjenigen, die in der Natur überlebt hatten und darum besonders glaubwürdig wirkten [1]. Mit der Entstehung der Sprache wurde das gesprochene und später das geschriebene Wort zum bedeutenden Vorteil im Kampf ums Überleben - Erfahrungen, die andere gemacht hatten, konnte von nun an jeder für sich nutzen [2]. Noch heute sind es die Eltern, die uns durch unsere Jugend führen, danach Freunde, Lehrer und berufliche Vorbilder, die uns leiten. Kurz: Der Rat der anderen ist uns wichtig.

\section{Von Eindrücken zu Zahlen}

Auch die Medizin lebt von Ratschlägen: Zunächst waren es grosse Ärzte, die im klinischen Alltag und in ihren Werken von den gemachten Erfahrungen berichteten. Anders als heute wurde vorwiegend selbst Erlebtes und von den eigenen Lehrern Erlerntes vermittelt [3]; die Reputation des Autors überzeugte, und weniger Zahlen und Statistik. Ja, noch im Jahre 1837 stritten sich in Paris Risueno d'Amador und Pierre Charles Alexandre Louis in der Académie Royale de Médecine, die Ludwig XVIII. 1820 ins Leben gerufen hatte, über die Frage, ob Medizin als Kunst oder als exakte Wissenschaft zu betrachten sei [4]. Die einen hielten jeden Patienten für einmalig und die Behandlung daher für einen Akt der Kreativität des jeweiligen Arztes; entsprechend verdammten sie quantitative Angaben über Patienten und Erkrankungen. «L'homme moyen n'existe pas!», war ihr Credo [5]. Pierre Charles Alexandre Louis hielt dagegen, dass wir nur lernen, wenn wir unserer Erfahrung Zahlen zuordnen, ihren Wert und ihr Gewicht bestimmen, ihre Veränderung beim Auftreten von Seuchen und Epidemien beschreiben, was zuletzt zur naturwissenschaftlichen Medizin führte, wie wir sie heute kennen [6].

Die Zahlen haben sich durchgesetzt: Heute sind es die Ergebnisse grosser Trials, die medizinisches Handeln beherrschen. Es sind nicht mehr einzelne Ärzte, vielmehr Guidelines Committees, Advisory Boards, Working Groups und Experten aller Art, die unser Handeln und unsere Entscheidungen bestimmen oder zu lenken suchen. Grundsätzlich ist dies zu begrüssen; wir wissen, dass unser Urteil, insofern es sich auf die tägliche Erfahrung stützt, nicht selten übereilig Ursachen ortet, wo es keine gibt, Spontanverläufe dem eigenen Tun zuschreibt und Einzelfälle überschätzt.
Bias - die schiefe Wahrnehmung, die sich im Alltag kaum vermeiden lässt - trübt unser aller klinisches Denken, wenn sie es auch nicht überflüssig macht. Gerade deshalb braucht es pathophysiologische Konzepte und die Ergebnisse verblindeter und randomisierter Versuche als Grundlage ärztlichen Handelns. Als Erster hatte 1948 Austin Bradford Hill, ein englischer Epidemiologe, den randomisierten klinischen Versuch verwendet und damit die Überlegenheit des Streptomycins gegenüber den bisher üblichen Liegekuren in der Behandlung der Lungentuberkulose belegt [7]. Die Entdeckung von Mykobakterien als Ursache der Tuberkulose durch Robert Koch und des Streptomycins durch Selman Waksman und Albert Schatz alleine genügten nicht. Erst diese strikte Prüfung neuer therapeutischer Verfahren in randomisierten Trials führte zur evidenz-basierten Medizin, wie wir sie heute kennen [8].

\section{Von Ratschlägen zu Guidelines}

Seither gründen sich Ratschläge in der Medizin wenn immer möglich auf Wissen und nicht alleine auf persönliche Erfahrung - in der globalen Welt nennt man sie Guidelines. Guidelines sind Leitlinien, die eine Richtung angeben - eine Richtung und nicht einen Weg. Sie versuchen, uns in unserem ärztlichen Tun zu leiten, sie sind kein Befehl von höherer Stelle; Ratschläge von Experten sind keine Gesetze, die es buchstabengetreu zu befolgen gilt, der gesunde Menschenverstand des Arztes bleibt weiterhin gefragt. Dennoch haben Guidelines einen Anspruch, der über einen gelegentlichen Rat hinausgeht: Sie beanspruchen, den gegenwärtigen Stand der ärztlichen Kunst zu vermitteln.

Doch wie läuft dies ab? Wie kann Wissen in Anweisungen umgemünzt werden? Einfach ist dies nicht: Wissen wir doch spätestens seit den alten Griechen [9] und besonders seit Kant [10], dass Tatsachenurteile und Maximen nicht das Gleiche sind. Und in der Tat: Aus dem Satz «Statine vermindern bei Patienten mit Hypercholesterinämie Tod, Myokardinfarkt und Hirnschlag um 30\%» lässt sich nicht direkt die Empfehlung ableiten: «Alle Patienten mit erhöhtem Cholesterin sollten ein Statin einnehmen». Warum nicht? Weil das eine einen Sachverhalt umschreibt, das andere eine Anleitung zum Handeln. Natürlich suchen wir ein wissenschaftliches Urteil als Grundlage von Empfehlungen, die Empfehlung selbst aber setzt voraus, dass die Massnahme in einem kulturell bestimmten 
Wertesystem als wertvoll genug erachtet wird, um sie umzusetzen [11]. Wir müssen also überzeugt sein, dass der Aufwand den Nutzen lohnt, dass die ökonomischen und medizinischen Kosten die erzielte Wirkung wert sind. Deshalb sind auch Empfehlungen nicht in allen Ländern und Kulturen gleich; Empfehlungen sind nicht unabhängig von dem Land, Gesundheitssystem und politischen Umfeld, in dem man praktiziert. Seit wir uns nicht mehr auf transzendente Werte stützen können, bestimmt - wie Jürgen Habermas es für alle gesellschaftlichen Fragen forderte [12] - ein rationaler Diskurs gleichberechtigter Bürger (oder eben Mediziner), der sich an vernünftige Argumente halten soll, die jeweils gültigen Werte. Gewiss, früher war es anders («God send his son and not a committee») [13], aber heute kommen wir ohne Arbeitsgruppen nicht mehr aus - die Expertokratie beherrscht auch die Medizin, seit sie zu erfolgreich wurde, um von einem Einzelnen gemeistert zu werden.
Dennoch gibt es weitere Einschränkungen: Guidelines sind krankheitsfokussiert und gehen nur beschränkt auf die Tatsache ein, dass viele Patienten multiple Diagnosen aufweisen [16]. Gerade deshalb müssen Guidelines von einem kompetenten Arzt umgesetzt werden; sie sind gerade nicht ein Kochbuch für Anfänger, sondern Handlungsanweisungen, die auf einen individuellen Patienten mit Umsicht und Erfahrung angepasst werden müssen.

Schliesslich kann man sich zu Recht fragen, ob Guidelines, die von ausländischen Expertengremien verfasst wurden, für die Patienten in der Schweiz zur Anwendung kommen sollen: Lässt sich die Schweizer Bevölkerung auf der Grundlage amerikanischer Statistiken behandeln? Und in der Tat ist beispielsweise das absolute Risiko, das in der Schweiz lebende Patienten mit einem bestimmten Blutdruck, Cholesterin- oder Glukosespiegel aufweisen, deutlich geringer als für solche aus Framingham oder dem Norden Europas.

\section{«Guidelines sind Empfehlungen aufgrund des gegenwärtigen Standes des Wissens. Sie werden aufgrund von Studienergebnissen erarbeitet, die den klinischen Alltag nur unvollständig abbilden - dennoch sind sie wichtige Ratgeber für Klinik und Praxis»}

\section{Vom Nutzen von Guidelines}

Braucht es daher Guidelines? Warum können wir nicht selbst entscheiden? Schliesslich haben wir ja alle studiert und unsere Erfahrungen gemacht. Und gewiss, die Aufklärung hat uns das Sapere aude - das «wage zu wissen»- gelehrt. Kant hat es noch deutlicher formuliert: Die Aufklärung ist der Ausgang des Menschen aus seiner selbstverschuldeten Unmündigkeit [14]. Sind wir nicht mündig genug, um selbst zu entscheiden? Hat die Aufklärung versagt? Unbestritten brauchen Schüler, Studenten, aber auch Assistenten, die sich in Weiterbildung befinden, Anleitungen für ihr Tun. Braucht auch der Facharzt diese Hilfe oder sind Guidelines ein unnötiges Diktat von Experten? Die Fortschritte der Medizin haben das Wissen in einem Mass erweitert, das es für den einzelnen Arzt unmöglich macht, noch alles so zu überblicken, um in jedem Fall die richtige Entscheidung zu fällen. Auch gehen Guidelines davon aus, dass sich praktische Richtlinien zur Abklärung und Behandlung von Krankheiten nur aus der verfügbaren wissenschaftlichen Erkenntnis ableiten lassen und nicht aus autistischen Erfahrungen [15] - daher die Legitimation der Experten.

Guidelines können den praktisch tätigen Ärzten in ihrem Alltag nützliche Hinweise geben; dies gilt namentlich für Guidelines zu Hypertonie, Diabetes, Hyperlipidämien oder Osteoporose. Richtlinien zu anderen Leiden kommen in der Hausarztpraxis selten zur Anwendung, spielen aber in Kliniken eine grosse Rolle.
Die lineare Beziehung zwischen Blutdruck, Cholesterin und Glukosespiegel bleibt sich aber - wenn auch auf etwas tieferem Niveau - gleich. Entsprechend hat die European Society of Cardiology ihre Risk Scores [17] für die nördlichen Länder sowie Mittel- und Südeuropa angepasst. Aktuelle Daten für die Schweiz sind allerdings nicht verfügbar. Es wäre deshalb wünschenswert, dass mehr schweizerische Daten auch von Patienten der Grundversorgung, ihrer Behandlung und ihrem Verlauf verfügbar würden; nur so lassen sich die Guide-

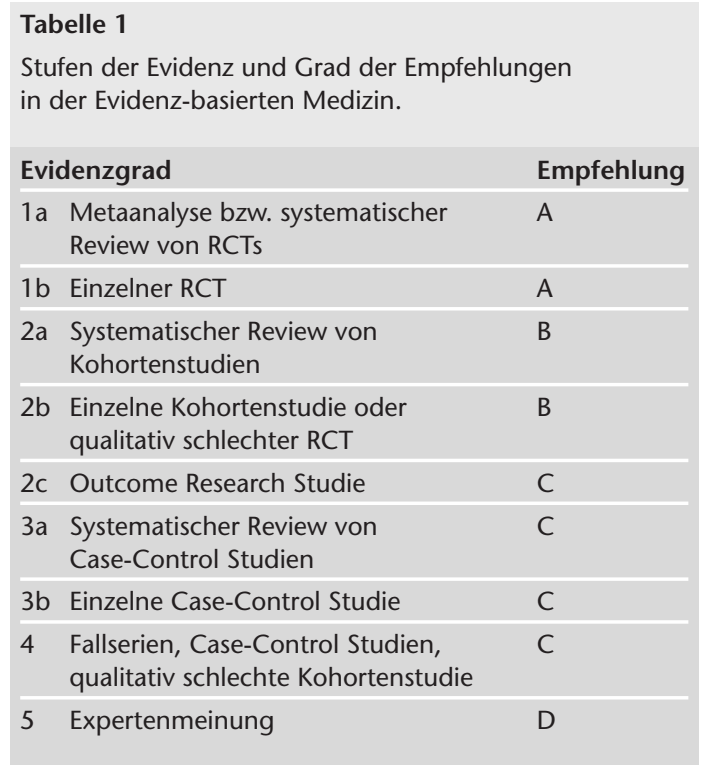




\section{Abbildung 1}

Stufen der klinischen Forschung von genetischer Information über Studien an Zellen, Organen bis zu Patienten und Populationen.

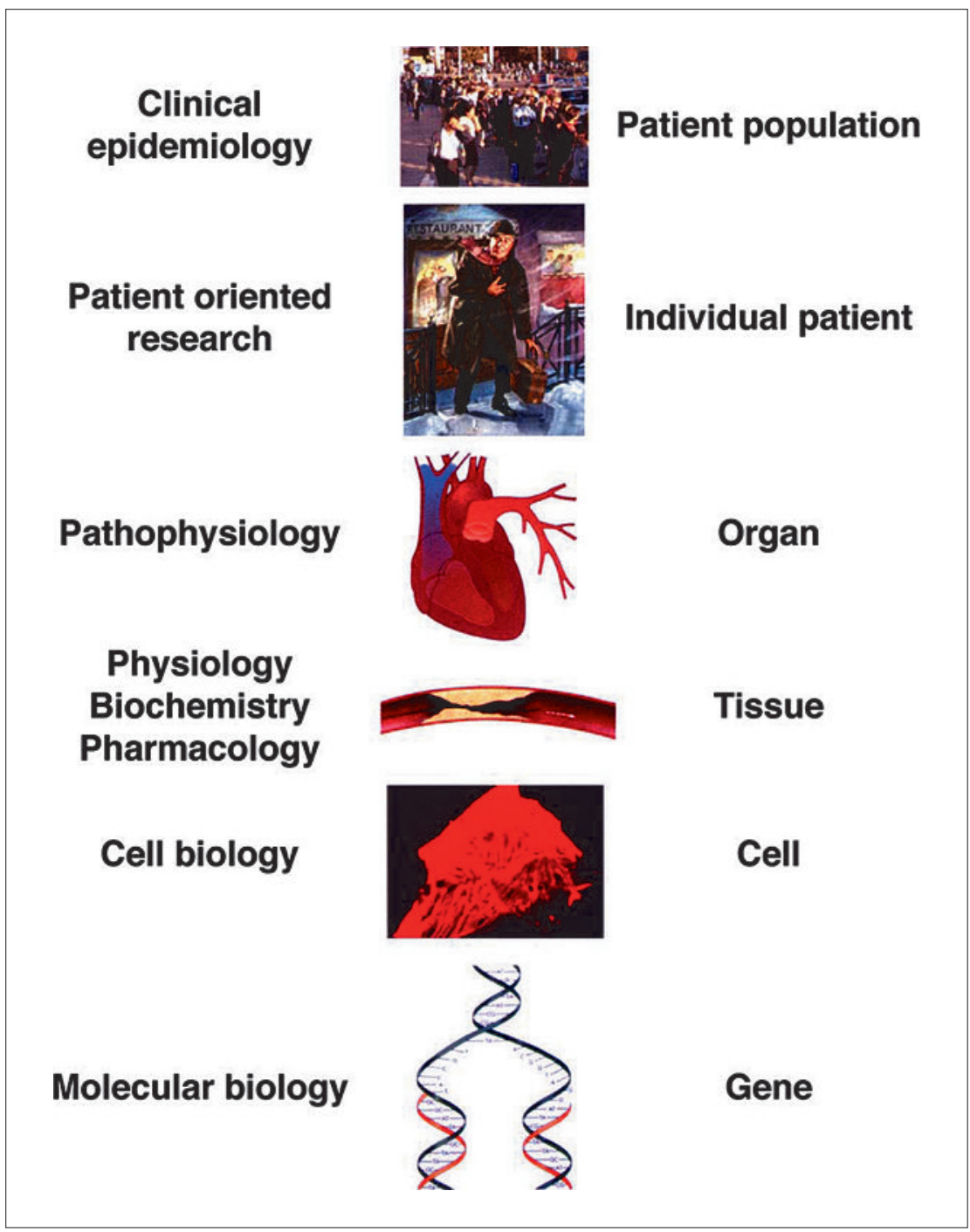

Abbildung 2

Stufen der Evidenz.

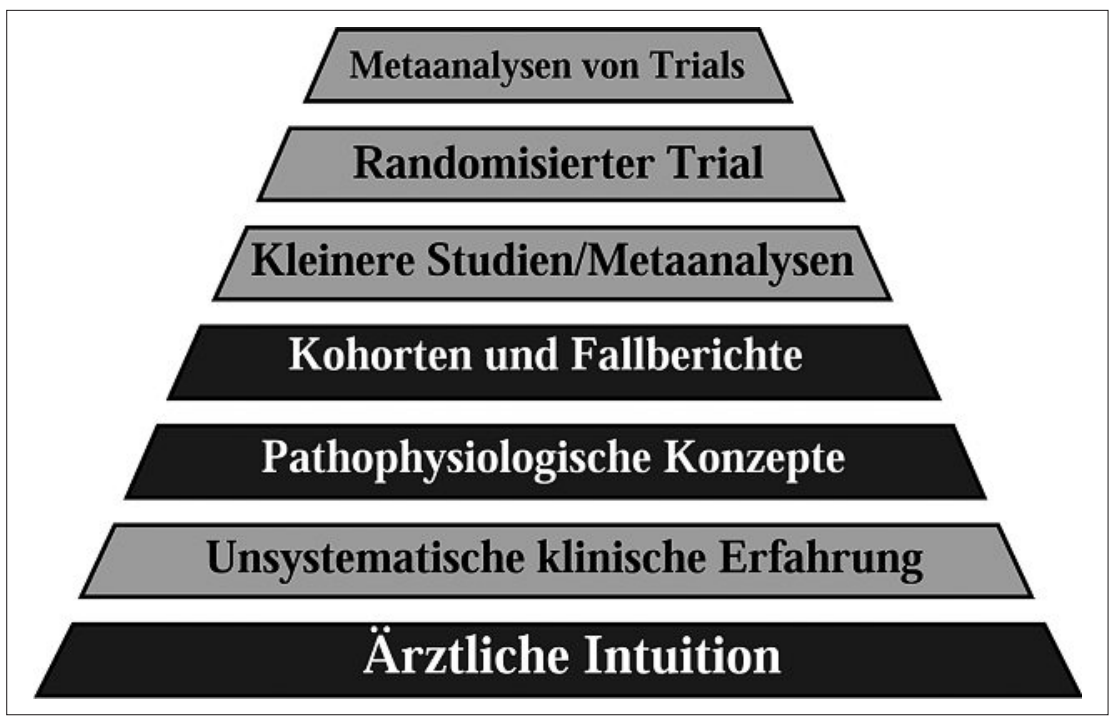

lines für die tägliche Praxis anpassen, weil auch Kosten-Nutzen-Überlegungen vom absoluten Risiko abhängen. Dazu bräuchte es aber vermehrt Forschung im Bereich Epidemiologie und Hausarztmedizin, vor allem auch entsprechende finanzielle Mittel.

\section{Das Gewicht der Evidenz}

Wir wissen nicht in allen Bereichen gleich viel - über die Behandlung des hohen Blutdrucks können wir mehr sagen als über den Umgang mit Patienten mit Amyotropher Lateralsklerose. Zunächst entsteht Wissen unter verschiedenen Bedingungen: in experimentellen Untersuchungen, Tiermodellen, Fallberichten, kleineren Proof-of-concept-Studien und schliesslich an grossen Patientenpopulationen (Abb. 1). Nicht alles ist gleichbedeutend; entsprechend hat man verschiedene Stufen von Evidenz festgelegt (Abb. 2). Am sichersten sind wir, wenn uns mehrere randomisierte Studien vorliegen; gelegentlich müssen wir uns mit kleineren Studien begnügen, aber bei vielen Leiden ist immer noch die eigene und publizierte Erfahrung aus Fallberichten das einzig Verfügbare - entsprechend werden die Empfehlungen gewichtet (Tabelle 1, S. 1692).

Damit ist es aber noch nicht getan: Jeder Arzt muss sich selbst fragen, ob und in welchem Masse sein Patient mit den Untersuchten übereinstimmt. Dazu muss man die Ein- und Ausschlusskriterien der grossen Studien kennen, die Demographie der eingeschlossenen Patienten; die Kompetenz des behandelnden Arztes bleibt daher auch im Zeitalter evidenz-basierter Medizin wichtig, ja, das Entscheiden wurde noch anspruchsvoller.

Verfügen wir auch über genügend Evidenz für eine optimale Betreuung unserer Patienten? Gewiss nicht, in vielen Bereichen ist die Evidenz dürftig bis gering; nicht nur bei seltenen Erkrankungen wie der FabryErkrankung oder der hypertrophen Kardiomyopathie, auch bei vielen neurologischen und allgemeinmedizinischen Leiden stützen wir uns mehr auf den gesunden Menschenverstand denn auf harte Daten. Sogar in der kardiovaskulären Medizin folgen wir den Empfehlungen der American Heart Association und dem American College of Cardiology, basieren noch fast die Hälfte der Richtlinien auf Evidenzgrad C (Tabelle 1) [18]. Weitere Forschung ist daher entscheidend, um die Versorgung von Patienten zu verbessern. Dies ist allerdings nur in einer konstruktiven Zusammenarbeit mit der forschenden Industrie im Rahmen der bestehenden Vorgaben und Gesetze möglich, da sonst die Mittel dazu fehlen. Eine Verteufelung dieser Zusammenarbeit macht daher keinen Sinn, ja gefährdet den Fortschritt der Medizin.

\section{Wer ist ein Experte?}

Guidelines Comittees sind Arbeitsgruppen von Experten, Ärzten und Forschern also, die über besonderes Wissen in dem zu bearbeitenden Gebiet verfügen. Doch wer ist ein Experte? Meist sind es Gesellschaften wie die European Society of Cardiology oder die World 
Gastroenterology Organisation, um nur einige zu nennen, oder Institute wie NICE (National Institute for Clinical Excellence) und andere mehr, die Guidelines herausgeben; sie bestimmen, wer Mitglied der Arbeitsgruppe wird und wer die Guidelines schreibt und schliesslich publiziert.

Ob die Experten wirklich Experten sind, lässt sich heute im PubMed oder unter www.isi.org bestimmen. Ob sie im Urteil auch frei und unabhängig sind, wie wir es uns wünschen, ist schwieriger zu erheben. Ohne Zweifel haben viele Experten Interessenkonflikte (oder Conflicts of interest im neuen Latein), denn sie sind gefragte Leute. Nicht nur Kollegen und staatliche Institutionen wollen von ihrem Wissen profitieren, auch die forschende Industrie setzt auf ihren Rat. Es gibt kaum einen erfolgreichen Kliniker oder Forscher, der nicht Mitglied in Advisory Boards wäre, Honorare für Vorträge oder Beiträge für Forschungsprojekte erhalten hätte. Nicht nur zeichnet ihn dies als erfolgreichen Mediziner aus, er braucht auch Forschungsgelder für seine eigene Tätigkeit. In der Psychiatrie ergab eine Studie, dass von 170 DSM panel members 95 finanzielle Beziehungen zur pharmazeutischen Industrie aufwiesen [19]. $\mathrm{Zu}$ berücksichtigen ist ferner, dass nicht nur finanzielle, vielmehr auch intellektuelle Conflicts unser Denken beeinflussen [20].

Schaffen diese Umstände ein Problem? Gewiss ist es denkbar, dass Mitglieder von Guidelines Committees, ja sogar die verantwortlichen Gesellschaften selbst, in ihrem Urteil von Unterstützungszahlungen, die sie in anderen Bereichen ihrer Tätigkeit von Herstellern empfohlener Produkte erhalten, beeinflusst werden bzw. durch negative Empfehlungen um diese Beiträge fürchten - Conflicts of interest von Mitgliedern solcher Kommissionen sind also durchaus ein Problem. Wie können wir dem begegnen? Transparenz gilt heute als geeignete Lösung, und so wurde die Angabe solcher Conflicts in den Acknowledgments solcher Dokumente ein Muss. Viel erreicht haben wir damit wohl nicht; nicht nur werden die bestehenden Konflikte unvollständig angegeben [21], Gewissheit, dass dadurch unabhängig vorgegangen wurde, entsteht so gerade nicht.

Dann ist die Art der Conflicts bedeutend; Experten mit Tätigkeiten mit vielen forschenden Firmen sind unabhängiger als solche, die nur einen Produzenten beraten. Ja, Experten mit Hang zu einer Firma sind als Mitglieder solcher Guidelines Committees nicht geeignet. Experten ohne jede Verbindung schliesslich wären wünschenswert, gehören in der Regel aber nicht zu den Besten ihres Fachs. Zuletzt müssen die Empfehlungen selbst überzeugen: Die Evidenz-basierte Medizin gründet sich auf Logik und klare Daten; was nachvollziehbar ist, ist überzeugend. Entsprechend sind solche Dokumente kritisch zu lesen.

\section{Beurteilung von Empfehlungen}

Somit müssen sich Empfehlungen dem Glaubwürdigkeitstest unterziehen: Was entscheidend ist, sind die berücksichtigten Studien (Ist die Evidenz vollständig?), ihre Bewertung (Sind sie randomisiert und doppelblind? Wurden sie unabhängig reproduziert?) und die daraus sich ergebende Empfehlung (Ist sie angemessen oder übertrieben?). Die Vollständigkeit der Evidenz kann durch Publication Bias beeinträchtigt sein: Negative Studien erblicken das Licht der Journals weniger also solche mit positiven Resultaten - schuld daran ist unser Hang zu Interessantem und derjenige der Herausgeber nach Aufmerksamkeit. So wurde beispielsweise ein Drittel der von der Federal Drug Administration registrierten Trials mit Antidepressiva nicht publiziert [22]. Auch der Umgang von Merck mit Daten zur kardiovaskulären Sicherheit von Rofecoxib sind ein Beispiel von Publication Bias [23]. Seit der Einführung eines Trial Registers durch die amerikanische Regierung (www.clinicaltrials.gov) und der Veröffentlichung von Design-Publikationen und Trial-Manualen [24] ist die Unterdrückung von Ergebnissen schwierig geworden. Dazu beigetragen hat auch die Initiative der Herausgeber wichtiger Journals, dass die Primary Investigators direkten Zugang zu allen Daten von Trials des Sponsors erhalten müssen. Auch der heutige Review-Prozess in angesehenen Zeitschriften mit erfahrenen Experten und einem statistischen Reviewer hat dafür gesorgt, dass die Qualität wissenschaftlicher Daten heute so gut ist wie nie zuvor. Die Conflicts einzelner Mitglieder solcher Arbeitsgruppen lassen sich durch Thematisierung des Problems zu Beginn der Arbeit, durch transparente Offenlegung und durch eine angemessene Zahl von Experten minimieren ausschliessen wohl kaum. Schliesslich ergibt auch der Vergleich mit Publikationen anderer Guidelines Committees Auskunft über den Kern der Aussagen: Sind alle Guidelines konsistent oder gibt es grosse Unterschiede? Zuletzt muss jeder Arzt selbst entscheiden, inwiefern die Empfehlungen in seinem Umfeld, im Gesundheitssystem, in welchem er arbeitet, umsetzbar sind oder nicht.

\section{Was zu bedenken bleibt}

Guidelines sind Empfehlungen aufgrund des gegenwärtigen Standes des Wissens. Sie werden aufgrund von Studienergebnissen erarbeitet, die den klinischen Alltag nur unvollständig abbilden - dennoch sind sie wichtige Ratgeber für Klinik und Praxis. Sie sind kritisch zu hinterfragen, inwiefern sie die verfügbare Evidenz angemessen berücksichtigen und inwiefern ihre Empfehlungen dadurch gestützt werden. Conflicts ihrer Autoren sind transparent zu machen und eine angemessene Zahl von beteiligten Experten in jeder Arbeitsgruppe soll sicherstellen, dass diese die Empfehlungen möglichst wenig beeinflussen. Zuletzt ist es aber die Prüfung der zugrundegelegten Evidenz und die Konsistenz der daraus abgeleitete Empfehlungsgrad, den es zu hinterfragen gilt - Transparenz alleine hilft uns nicht weiter, selbständiges Denken bleibt uns auch im Zeitalter von Guidelines nicht erspart. 


\section{Literatur}

1 Canetti E. Masse und Macht.

München: Carl Hanser; 1960. S. 267 ff.

2 Lüscher TF. Gedankenmedizin. Heidelberg: Springer; 2009. S. 101-110.

3 Heberden W. Some account of a disorder of the breast. Medical Transactions of the Royal College of Physicians of London. 2, 59-67, 1772.

4 Lüscher TF. Gedankenmedizin. Heidelberg: Springer; 2009. S. 77-86.

5 Mathews JT. Quantification and the quest for medical certainty. Ewing, NJ, USA: Princeton University Press; 1995. S. 14-38.

6 Haynes RB, Sackett D, Guyatt GH, Tugwell P. Clinical epidemiology: How to do clinical practice research. $3^{\text {rd }}$ edition. Philadelphia: Williams and Wilkins; 2005.

7 Hill AB. Streptomycin treatment of pulmonary tuberculosis: A Medical Research Council investigation. Brit. Med. J. 1948:769-773.

8 Yusuf S, Cairns JA. Camm J, Fallen EL, Gersh BJ: Evidence-based Cardiology, London: BMJ Books; 2003.

9 Aristoteles. Nikomachische Ethik. Zürich: Artemis \& Winkler; 2001.

10 Kant I: Die drei Kritiken. Stuttgart: Alfred Kröner Verlag, S. 1069.

11 Lüscher TF. Gedankenmedizin. Heidelberg: Springer; 2009. S. $81 \mathrm{ff}$

12 Habermas J. Theorie des kommunikativen Handelns. Frankfurt: Suhrkamp; 1987.

13 Vanhoutte PM.

Persönliche Mitteilung 1987.
14 Kant I. Was ist Aufklärung? Hamburg: Meiner Philosophische Bibliothek; 1999, S. 20.

15 Bleuler E. Das autistisch-undisziplinierte Denken in der Medizin und seine Überwindung. Saarbrücken: Verlag Classic Edition; 2009.

16 Shaneyfelt TM, Centor RM. Reassessment of clinical practice guidelines - Go gently into that good night. J. Amer. Med. Ass. 2009;301: 868-869.

17 ESC Pocket Guidelines on Cardiovascular Prevention. European Society of Cardiology, Sophia-Antipolis, 2007.

18 Tricoci P, Allen JM, Kramer JM, Califf RM, Smith SC. Scientific evidence underlying the ACC/AHA clinical practice guidelines. J. Amer. Med. Ass. 2009;301:831-841.

19 Cosgrove L, Krimsky S, Vijayaraghavan M, Schneider L. Financial ties between DSM-IV panel members and the pharmaceutical industry. 2006;75:154-160.

20 Lüscher TF: Conflicts of Interest oder Interesse am Konflikt? Schweiz Ärztezeitung. 2001;82:2137-2144.

21 Okoke K, Kocher MS, Wie EX, Mehlman CT, Bhandari W: Accuracy of conflict-of-interest disclosures reported by physicians. New Engl J Med. 2009;361:1466-1474

22 Turner EH, Matthews AM, Linardatos E, Tell RA, Rosenthal R. Selective publication of antidepressant trials and its influence on apparent efficacy. NEJM 2008;358:252-260.

23 Topol E: Failing the public health - Rofecoxib, Merck and the FDA. NEJM 2004;351:17-19.

24 Kloner RA, Birnbaum Y. Cardiovascular trials review, $10^{\text {th }}$ edition, Darien, CT, USA: Le Lacq Communication; 2006. 